\title{
Spatial Organization of Functional Groups on Bioactive Supramolecular Glycopeptide Nanofibers for Differentiation of Mesenchymal Stem Cells (MSCs) to Brown Adipogenesis
}

\author{
Ozum S. Caliskan, Melis Sardan Ekiz, Ayse B. Tekinay,*(i) and Mustafa O. Guler*(1) \\ Institute of Materials Science and Nanotechnology, National Nanotechnology Research Center (UNAM), Bilkent University, 06800 \\ Ankara, Turkey
}

\section{Supporting Information}

\begin{abstract}
Spatial organization of bioactive moieties in biological materials has significant impact on the function and efficiency of these systems. Here, we demonstrate the effect of spatial organization of functional groups including carboxylate, amine, and glucose functionalities by using self-assembled peptide amphiphile (PA) nanofibers as a bioactive scaffold. We show that presentation of bioactive groups on glycopeptide nanofibers affects mesenchymal stem cells (MSCs) in a distinct manner by means of adhesion, proliferation, and differ-

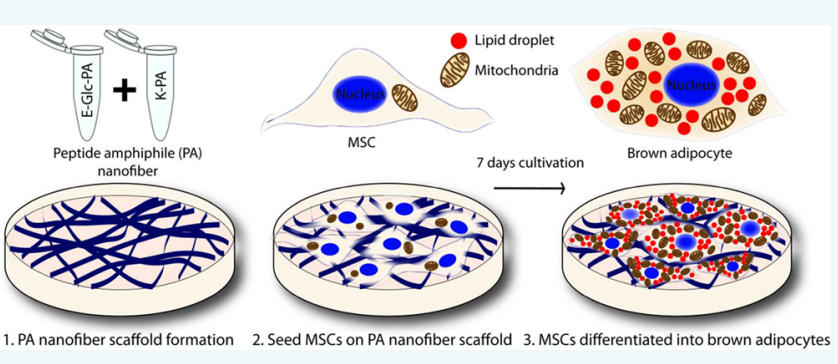
entiation. Strikingly, when the glutamic acid is present in the glycopeptide backbone, the PA nanofibers specifically induced differentiation of MSCs into brown adipocytes in the absence of any differentiation medium as shown by lipid droplet accumulation and adipogenic gene marker expression analyses. This effect was not evident in the other glycopeptide nanofibers, which displayed the same functional groups but with different spatial organization. Brown adipocytes are attractive targets for obesity treatment and are found in trace amounts in adults, which also makes this specific glycopeptide nanofiber system an attractive tool to study molecular pathways of brown adipocyte formation.
\end{abstract}

\section{INTRODUCTION}

Spatial organization of bioactive groups in biological materials is important in function and mechanism of cellular events. ${ }^{1,2}$ The peptide amphiphile (PA) molecules are a versatile class of materials that can self-assemble into a variety of bioactive nanostructures. The PAs integrate structural features of amphiphiles with functions of peptides that can be biologically active. ${ }^{3}$ The PAs can be designed for different tasks, as they have tailorable bioactivity depending on the peptide sequence, which can be used in diverse biomedical applications including regenerative medicine and drug delivery. ${ }^{4-11}$ Self-assembly of PA molecules can be triggered by electrolyte addition, changing $\mathrm{pH}$, or coassembling with oppositely charged systems. ${ }^{\text {12,13 }}$ Through self-assembly, the PAs can form high-aspect-ratio nanofibers, which are powerful and exclusive tools for fabrication of scaffolds due to their unique bioactive extracellular matrix (ECM) mimetic properties, which direct cellular responses and cell fate., ${ }^{3,4,14}$ In addition, the PA nanofibers present bioactive peptide epitopes with a much higher density compared to single bioactive peptide molecules, ${ }^{3,15}$ and high density epitope presentation is known to strongly enhance the activity of bioactive sequences. Spatial organization of functional groups on PA nanofibers affects induction of biological mechanisms, and understanding the factors affecting bioactivity of PA nanofibers is vital for the successful engineering of supramolecular architectures with desired property and function and for better understanding of the biological processes. Although there are a few studies showing the advantages of spatial organization of peptide functionalities in two dimensions, ${ }^{3,16}$ the effect of supramolecular spatial organization has not been addressed in detail.

In this study, we show the influence of supramolecular spatial organization of bioactive groups in PA nanofibers in cellular processes. For this purpose, the PA nanofiber systems were designed with bioactive glycopeptide amphiphiles. Bioactivity of glucose residues has been shown by investigating the roles of glycosylation in proteins and peptides, the roles of oligosaccharides attached to cell surface, or influence of glycosaminoglycans in the extracellular matrix (ECM) for cell adhesion and differentiation. ${ }^{17-19}$ Glucose unit conjugated to a serine amino acid side chain was used as a bioactive group in the PA system. Two different amino acids were used as flanking amino acid: glutamic acid and lysine residues for E-Glc-PA and K-Glc-PA, respectively (Figure 1). Flanking amino acid refers to the amino acid type that is located around the bioactive sequences. The K-PA and E-PA molecules lack the bioactive glucose units. The E-Glc-PA was mixed with K-PA and K-GlcPA was mixed with E-PA and two nanofiber systems with the same amino acid composition and the same number of bioactive groups were obtained; however, as supramolecular

Special Issue: Peptide Conjugates for Biological Applications

Received: November 1, 2016

Revised: December 5, 2016

Published: December 8, 2016 

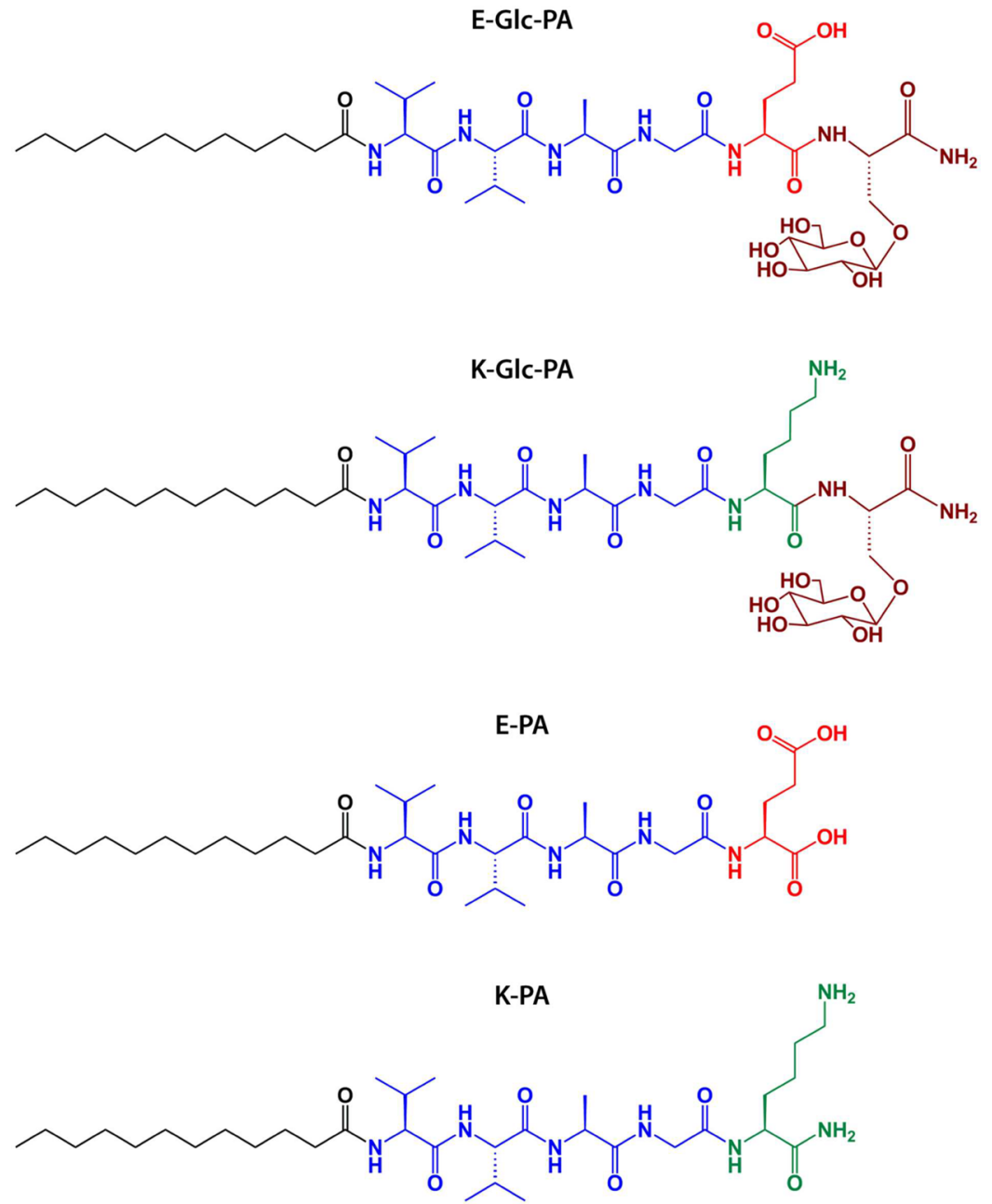

Figure 1. Chemical structures of the peptide amphiphiles. Color codes of the structures indicate alkyl tail (black), $\beta$-sheet forming amino acids (blue), positively charged amino acids (green), negatively charged amino acids (red), and glucose conjugated serine amino acid (dark red).

systems, the spatial organizations of the bioactive groups were distinct in these two systems. Mixture of K-PA and E-PA was used as a control for testing the bioactivity of glucose residues on cellular responses. Rat mesenchymal stem cells (MSCs) were cultured on the PA nanofiber scaffolds, and the effect of spatial organization of functional groups on cellular responses of MSCs was studied. Adhesion and proliferation of the cells exhibited different profiles between the E-Glc-PA/K-PA and KGlc-PA/E-PA surfaces. We further observed that the E-Glc-PA/ K-PA nanofiber system is a striking inducer of brown fat adipogenesis of MSCs, which was significantly much stronger than the K-Glc-PA/E-PA and other control surfaces. This result reported the importance of spatial organization of functional groups in the self-assembled PA systems and demonstrated that different conformational signals create a significant difference on cell behavior of MSCs. In addition, this study introduced a novel scaffold to culture stem cells in order to trigger differentiation into brown fat cells. Brown fat cells are in the focus of obesity related studies; however there is limited access to brown fat cells because they are rarely found in the body. ${ }^{20,21}$ Thus, our results also provide a method for obtaining brown fat cells from stem cells with high yield.

\section{RESULTS}

Synthesis and Characterization of Peptide Amphiphile Molecules. The E-Glc-PA (lauryl-VVAGES $(\beta$-D-Glc)$\mathrm{Am})$ and K-Glc-PA (lauryl-VVAGKS $(\beta$-D-Glc)-Am) molecules were designed as glycopeptide amphiphiles (Figure 1). The KPA (lauryl-VVAGK-Am) and E-PA (lauryl-VVAGE) were synthesized to form supramolecular nanofibers with the glycopeptides through coassembly, forming two distinct nanofiber systems with the same number of functional groups and amino acids (Figure 1). The coassembly of K-PA and E-PA was formed as a non-glucose bearing PA nanofiber control. The PA molecules are composed of a hydrophobic alkyl tail (lauryl moiety), a $\beta$-sheet forming amino acid sequence (-VVAG-), and a charged amino acid for solubility and charge control. ${ }^{12}$ Glucose bearing PA molecules (K-Glc-PA and E-Glc-PA) have additional Ser-linked $\beta$-D-glucose glycoamino acid as bioactive unit (Figure 1 and Table $S 1$ in Supporting Information). The E-Glc-PA and E-PA bear a negative charge due to glutamic acid (E) residue, and K-Glc-PA and K-PA bear a positive charge due to lysine $(\mathrm{K})$ residues. All PA molecules were synthesized by solid phase peptide synthesis method, purified by preparative high performance liquid chromatography (HPLC), and characterized by liquid chromatography-mass spectrometry (LC-MS). 
PA Nanofiber Characterization. The oppositely charged PA molecules were mixed and characterized with scanning electron microscopy (SEM) (Figure 2A). SEM images showed

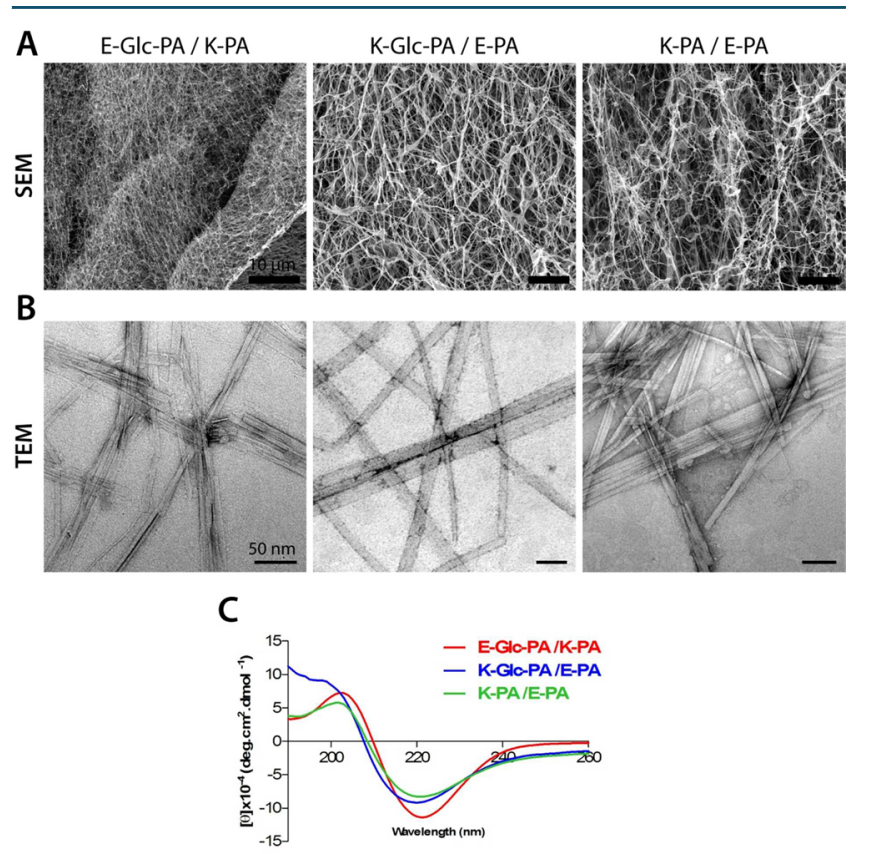

Figure 2. Morphological and structural properties of the peptide amphiphile nanofibers. (A) SEM images show ECM-like structure of PA nanonetworks. (B) TEM images (left and right) and STEM image (middle) show PA nanofibers. (C) Circular dichroism reveals the secondary structure of PA nanofibers.

that PA nanonetworks were porous and composed of nanofibers, which highly resemble the native ECM found around the cells. This resemblance makes the PA nanofibers a suitable microenvironment for the cells to attach, live, and proliferate. The closer visualization of PA nanofibers was performed by transmission electron microscopy (TEM) or scanning transmission electron microscopy (STEM). TEM and STEM images revealed that ECM-like structure was formed by high-aspect-ratio nanofibers with diameter of $8-10 \mathrm{~nm}$ and lengths reaching several micrometers (Figure 2B). Circular dichroism (CD) analyses were performed to understand the secondary structure of PA nanostructures formed upon mixing oppositely charged PA molecules. The CD spectra revealed that mixed PA systems show $\beta$-sheet structure, displaying a minimum around $220 \mathrm{~nm}$ and a maximum around $204 \mathrm{~nm}$ (Figure 2C). ${ }^{7,22}$ The PA molecules at neutral $\mathrm{pH}$ showed disordered conformation, except E-Glc-PA (Figure S2) molecule, which showed $\beta$-sheet indicating $\mathrm{CD}$ peaks. ${ }^{22}$ Overall, all three nanofiber systems (E-Glc-PA/K-PA, K-GlcPA/E-PA, and K-PA/E-PA) showed similar morphologies and molecular organizations.

Early Cellular Response on the PA Networks: Viability, Adhesion, and Proliferation. Biocompatibility of the PA nanofiber systems was assessed by monitoring the viability of cells cultured on the nanofiber networks. Cellular viability was checked after $12 \mathrm{~h}$ of incubation on the PA nanonetworks in order to discriminate the interference of proliferation on viability results. Viability results showed that all PA nanofiber systems were biocompatible for MSCs, comparable to uncoated tissue culture plates (Figure 3A).
A

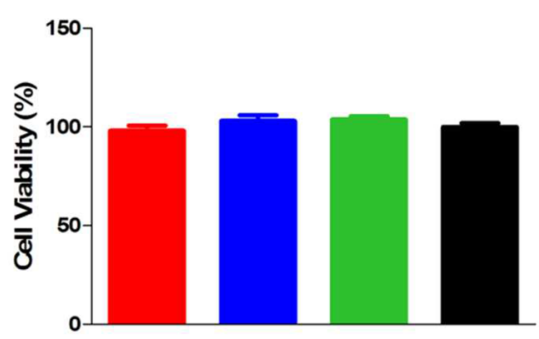

B

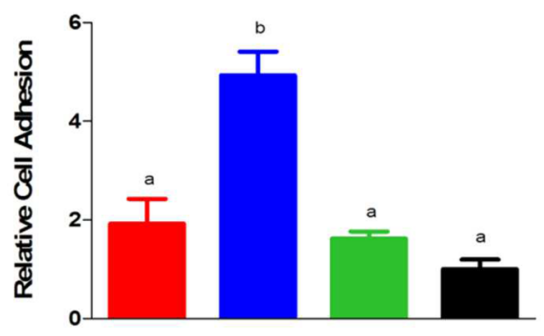

C

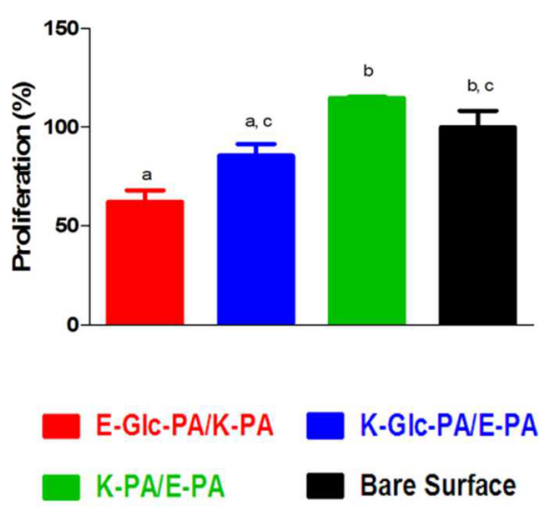

Figure 3. Early cellular responses on peptide nanofiber networks. (A) Cell viability on peptide nanofiber networks compared to bare surface, after $12 \mathrm{~h}$ cultivation period. (B) Number of adhered cells on different peptide nanofiber networks $5 \mathrm{~h}$ after cell seeding, in proportion to number of adhered cells on bare surface. (C) Proliferation rate of cells on different peptide nanofiber networks compared to bare surface, after $48 \mathrm{~h}$ cultivation period. Different letters denote significance at $p<$ 0.05

Adhesion behavior of cells on peptide nanofiber network systems is an important initial sign of bioactivity. Relative adhesion data were obtained by normalization of total number of adhered cells on peptide nanofiber networks to total number of cells on bare surface, which was bare tissue culture plate. Total number of cells on bare surface was accepted as one, and cell amount in other groups were normalized to cells on bare surface. Results showed that $5 \mathrm{~h}$ after cell seeding, cells adhered more on peptide nanofiber networks compared to bare surface (Figure 3B). This result indicated that PA nanofiber networks provide a favorable environment for cells to adhere. The first significant effect of supramolecular spatial organization of functional groups was also observed during adhesion assay. The number of cells adhered on K-Glc-PA/E-PA was significantly higher than the E-Glc-PA/K-PA, although both nanofiber systems contained the same functional bioactive groups in equal amounts. 

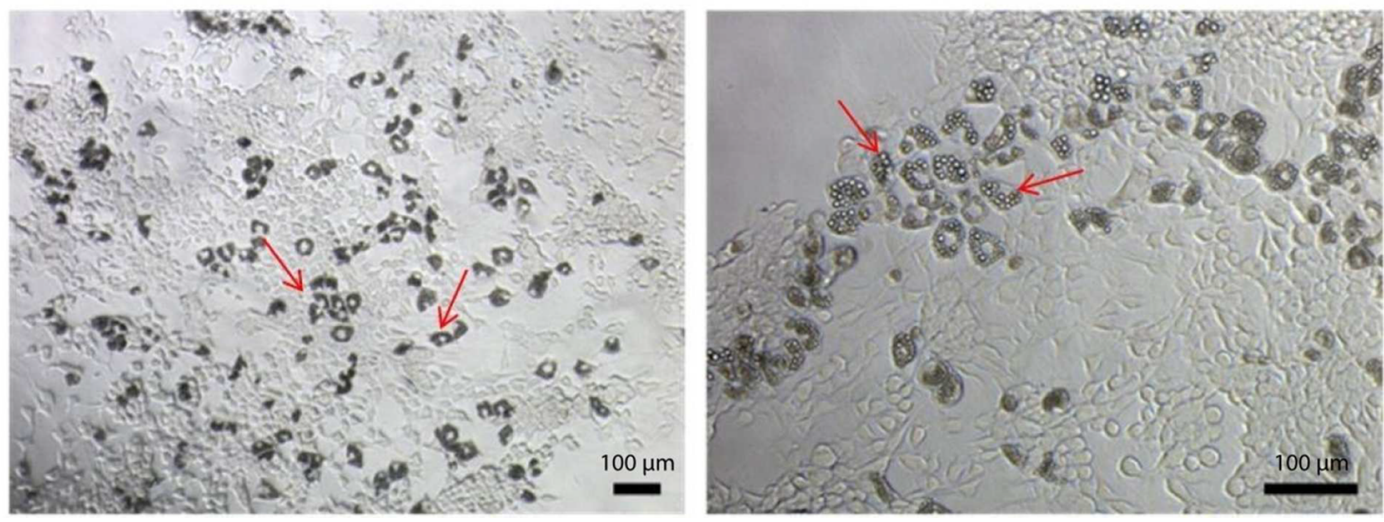

Figure 4. Lipid droplet formation by mesenchymal stem cells. Optical microscope images of rMSCs cultured on E-Glc-PA/K-PA nanonetwork with different scales, after 7 days of incubation. Darker areas are putative lipid droplets.

Effect of culturing on the PA nanofibers on proliferation profile of MSCs was studied by BrdU incorporation assay after $48 \mathrm{~h}$ of incubation. When compared to cells cultured on tissue culture plate, proliferation percentages of MSCs were found to be $62 \%$ for E-Glc-PA/K-PA, $85.54 \%$ for K-Glc-PA/E-PA, and $114.8 \%$ for K-PA/E-PA group (Figure 3C). Although E-Glc$\mathrm{PA} / \mathrm{K}-\mathrm{PA}$ and K-Glc-PA/E-PA nanofibers were observed to affect the proliferation at different levels, this difference was not statistically significant (difference between the two groups, $23 \%)$.

Investigation of the Differentiation Response of MSCs to Glucose Bearing Nanofiber Scaffolds with Different Spatial Organizations. Morphological changes of the cells that were seeded on PA nanofiber network groups were tracked in order to observe any changes triggered by different PA networks. Initially, accumulation of lipid droplet-like structures was observed in E-Glc-PA/K-PA system (Figure 4), whereas they were rarely observed in other groups. ${ }^{23}$ Therefore, investigation of the influence of different spatial organizations of PA nanofibers on MSC differentiation was specifically focused on adipogenesis.

Oil red-O is a fat-soluble diazol dye and stains neutral lipids such as triglycerides, diacylglycerols, and cholesteryl esters but not polar lipids (i.e., phospholipids, sphingolipids, and ceramides). ${ }^{24,25}$ Oil red-O staining demonstrated that MSCs accumulated lipid droplets in significantly high numbers when cells were cultured on E-Glc-PA/K-PA nanofiber network after 7 days of culturing (Figure 5A). On the other hand, the number of cells having lipid droplets and amount of total lipid droplets in K-Glc-PA/E-PA and E-PA/K-PA combinations and bare surface were very few compared to E-Glc-PA/K-PA group.

To better evaluate the differentiated cells, gene expression profiles of MSCs cultured on peptide nanofiber networks and on bare surfaces were analyzed. Expression levels of two widely used adipogenesis markers adiponectin (ADIPOQ) $)^{26-29}$ and fatty acid binding protein 4 (FABP4 $)^{30-33}$ were examined. On day 7 , a remarkable increase in expression of these adipogenesis gene markers was observed for E-Glc-PA/K-PA compared to three other groups (Figure $5 \mathrm{~B}$ ). There was a slight increase in expression of both genes on K-Glc-PA/E-PA group compared to K-PA/E-PA and bare surface groups. However, this increase was much less than the one that was observed on E-Glc-PA/KPA. In terms of gene expressions, the K-PA/E-PA and bare surface groups were comparable for both genes on both days. This result showed that the glucose has to be included in peptide nanofiber system in order to obtain this specific bioactivity. Overall mRNA expression results support the lipid droplet formations which were observed by oil red-O staining.

Oil red-O staining, adiponectin, and FABP4 mRNA expression analyses were also performed on day 11 in order to track the trend observed on day 7 . The results were similar on both experimental days. Day 11 oil red-O staining underlines the importance of spatial organization of PA nanofibers. E-Glc-PA/K-PA and K-Glc-PA/E-PA had significantly different effects of on MSC adipogenesis (Figure S3). Like oil red-O, the trend in relative change of adiponectin and FABP4 gene expressions on day 7 and day 11 was also comparable to each other (Figure 5B), where E-Glc-PA/K-PA group had a strikingly higher level of expression.

Determination of the Type of Fat Cells Which Were Differentiated from MSCs on E-Glc-PA/K-PA Nanofiber Scaffolds. There are mainly two types of different fat cells, white and brown fat cells. ${ }^{34}$ White adipocytes contain single or a few large lipid droplets that appear to comprise the majority of cell volume. The cytoplasm and nucleus of white adipocytes are found at the cell periphery. ${ }^{34,35}$ Brown adipocytes, on the other hand, are characterized by small and multilocular lipid droplets. ${ }^{36}$ The type of fat cells committed from MSCs on EGlc-PA/K-PA nanofiber systems was further investigated by staining with Nile red and TO-PRO-3, and stained cells were imaged with a high resolution confocal microscope. Nile red is a hydrophobic and fluorescent dye that selectively stains the intracellular lipid droplets. ${ }^{37}$ TO-PRO-3 sensitively stains the double stranded nucleic acids and is widely used as a nuclear counterstain. ${ }^{38}$ Most of the cells cultured on the E-Glc-PA/KPA nanofiber formed multilocular lipid droplets (Figure 6A). Multilocular lipid droplets were observed throughout the cytoplasm. Multilocular lipid droplets are a characteristic feature of brown fat cells, and other scaffolds did not induce high amount of lipid droplets (Figure S4).

Leptin and UCP1 are characteristic proteins of white and brown fat, respectively. ${ }^{39,40}$ Uncoupled protein 1 (UCP1), also called thermogenin, is a mitochondrial protein, which is uniquely expressed by the mitochondrion-rich brown adipocytes, and its function is to uncouple respiration from ATP synthesis. ${ }^{41-46}$ Leptin is an endocrine hormone, which is primarily produced by adipose tissue and is well-known as a satiety factor. ${ }^{47}$ Leptin is also determined as a marker gene to distinguish white fat cells and brown fat cells. ${ }^{39,40,48}$ Therefore, expression levels of leptin and UCP1 genes of MSCs cultured 


\section{A}
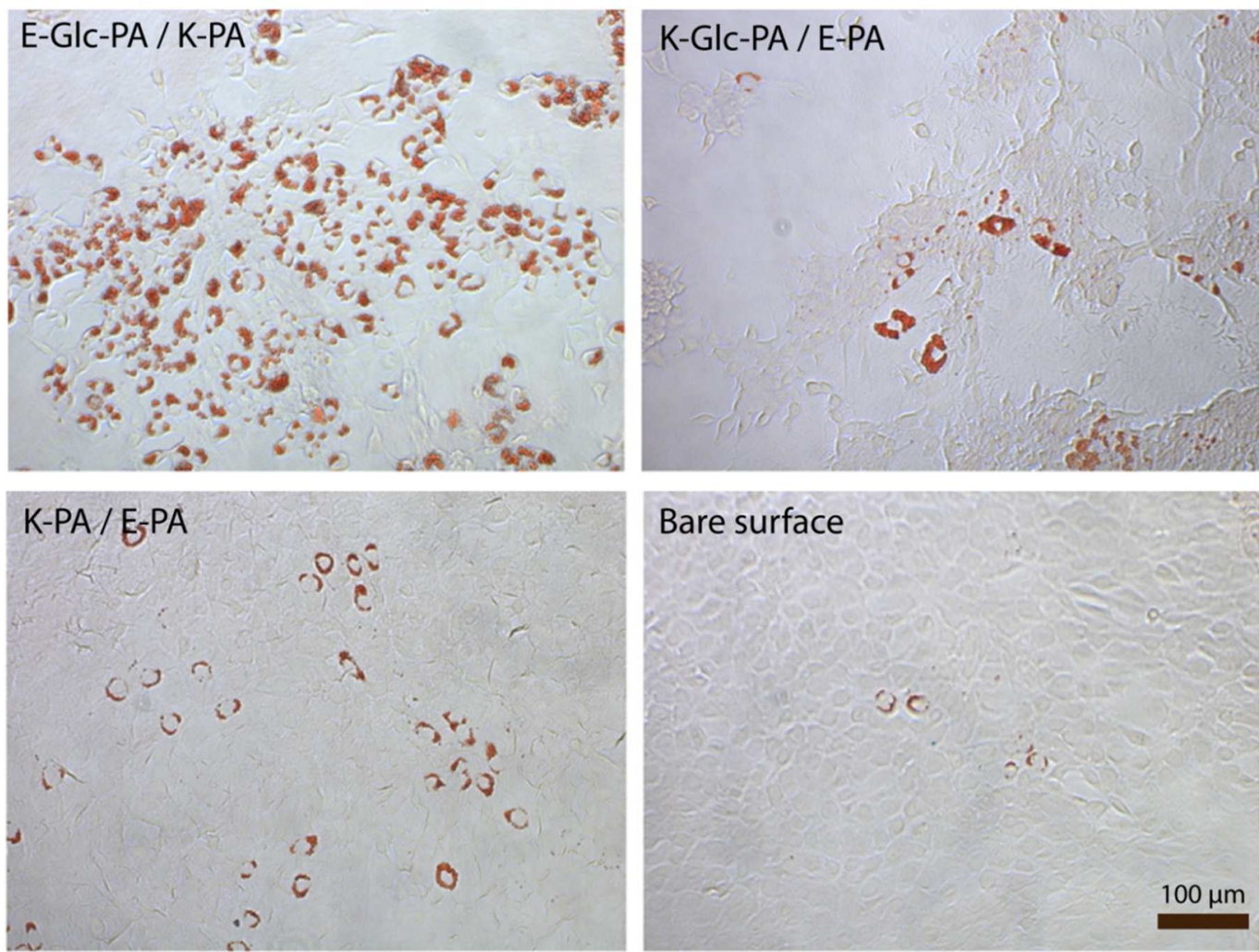

B

Adiponectin

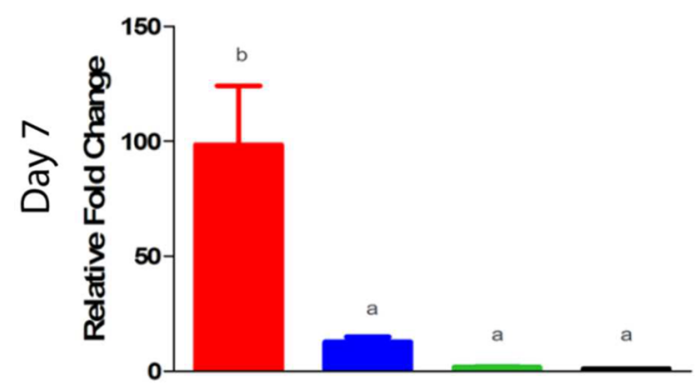

Adiponectin

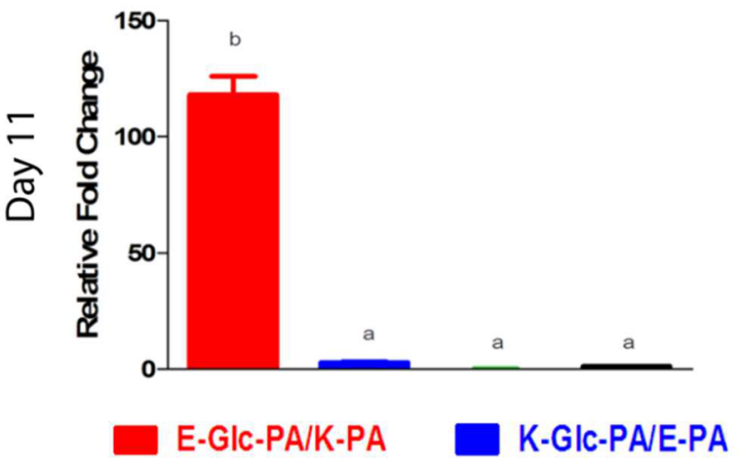

FABP4

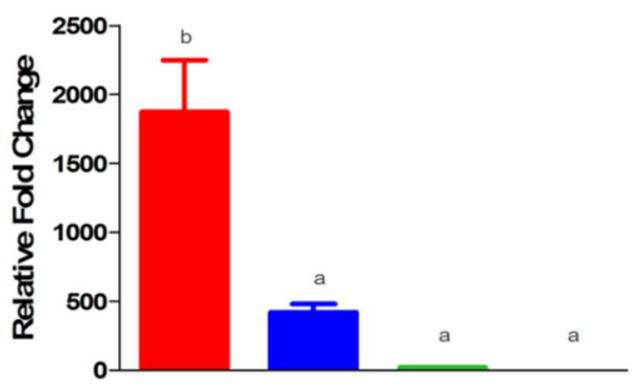

FABP4

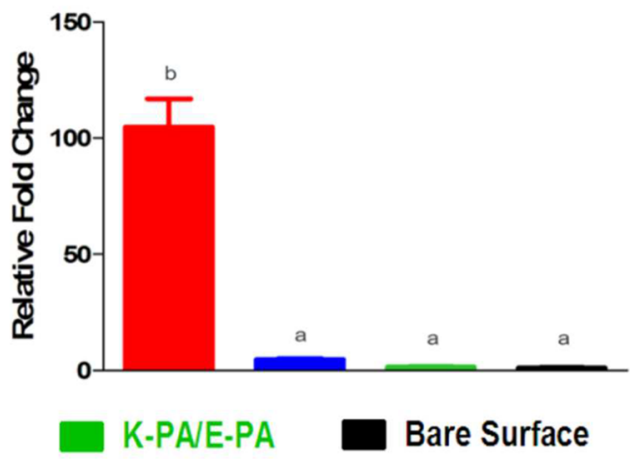

Figure 5. Determination of adipogenesis. (A) Oil red-O staining of cells cultured in maintenance medium for 7 days, on different networks, showing the lipid droplet accumulation. Red colors indicate lipid droplets inside cells. Scale bar is $100 \mu \mathrm{m}$ and equal for all images. (B) Expression analyses of adipogenesis marker genes, adiponectin and FABP4, in mesenchymal stem cells cultured in maintenance medium for 7 and 11 days. The expression level of each gene was normalized against GAPDH and bare surface. Different letters denote significance at $p<0.05$. 
A

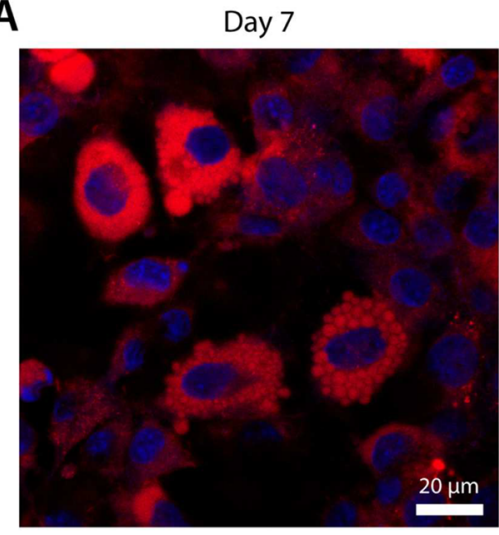

B

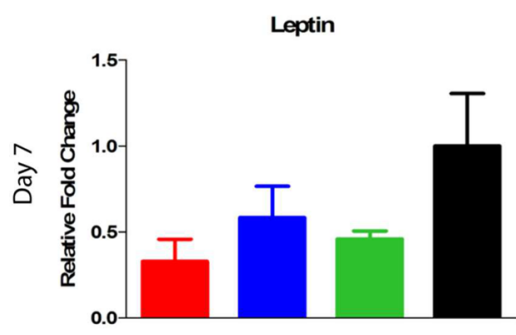

Leptin

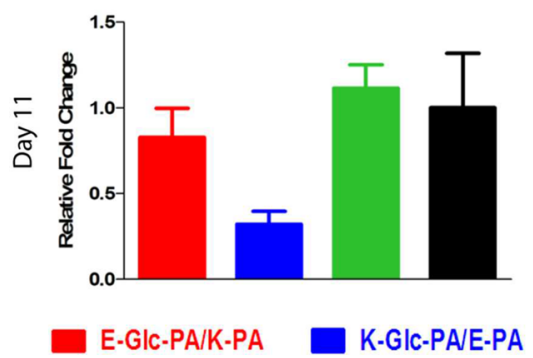

Day 11

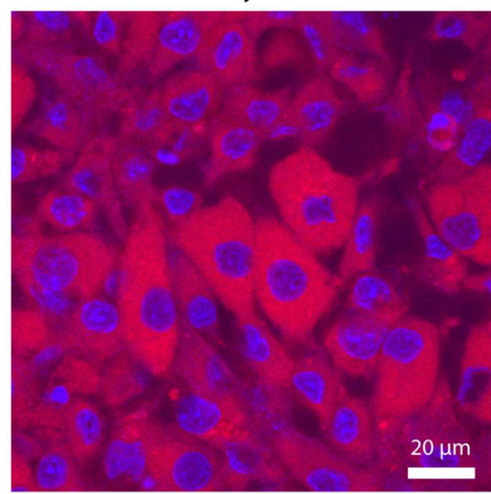

UCP1

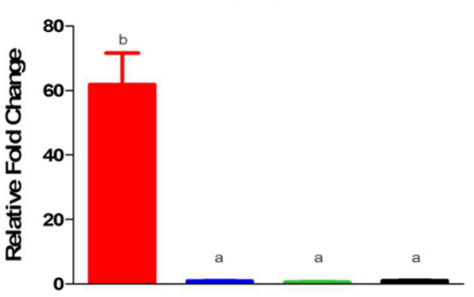

UCP1

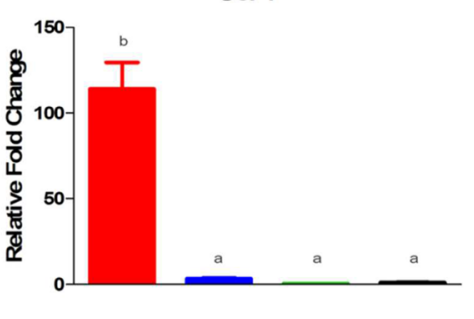

K-PAIE-PA $\square$ Bare Surface

Figure 6. Brown adipogenesis of stem cells cultured on E-Glc-PA/K-PA network. (A) Nile red (red color, lipid droplets) and TO-PRO-3 (blue color, nuclei) staining of mesenchymal stem cells cultured on E-Glc-PA/K-PA network, after 7 and 11 days of incubation in maintenance medium. Multilocular lipid droplets, as formed by brown fat cells, were clearly observed (magnification, 63X). (B) White fat marker gene, leptin, and brown fat marker gene, UCP1, expressions of mesenchymal stem cells cultured in maintenance medium for 7 and 11 days. Different letters denote significance at $p<0.05$.

on PA nanofiber scaffolds were analyzed. Results revealed that while leptin expression levels were not significantly different from each other for all experimental groups, expression level of UCP1 on the E-Glc-PA/K-PA nanofibers was remarkably high compared to K-Glc-PA/E-PA system and the control groups (Figure 6B). Variation in leptin levels among the different experimental groups was not more than 0.7 -fold change on both day 7 and day 11. The expression levels of UCP1 on KGlc-PA/E-PA, K-PA/E-PA, and bare surface were comparatively in the same range on day 7 and day 11, compared to the bare surface. On the other hand, expression of UCP1 on E-Glc$\mathrm{PA} / \mathrm{K}-\mathrm{PA}$ on day 7 was 61.8 -fold more than the bare surface and was also more than all other groups. UCP1 expression of cells cultured on E-Glc-PA/K-PA further increased on day 11, with a prominent 114-fold change compared to bare surface level. UCP1 expression analysis itself is striking in showing the importance of spatial organization.

\section{DISCUSSION}

The bioactive PA molecules with opposite charges selfassemble into nanofiber networks. The self-assembly process is driven by hydrophobic collapse, electrostatic interactions, and $\beta$-sheet formation, and the nanofibers are stabilized by van der Waals and hydrophobic forces and hydrogen bonding. ${ }^{49}$ SEM and TEM imaging showed that PA molecules create ECM-like highly porous environment formed by nanofibers. Circular dichroism spectroscopy revealed that coassembly of PAs has $\beta$ sheet secondary structure due to the incorporation of $\beta$-sheet driving amino acids into the PA backbone.

Biocompatible PA nanofibers exhibited different capacities to alter cell adhesion and proliferation among different PA coassembly combinations. Higher number of cells adhered on nanofiber scaffolds compared to bare surface indicating that PA niche provides more adhesion epitopes than that of tissue culture plate. The number of adhered cells was significantly higher on glucose-containing nanofiber systems compared to EPA/K-PA system, which shows the importance of glucose 
residue for cell adhesion. On the other hand, cellular adhesion on E-Glc-PA/K-PA was significantly less than K-Glc-PA/E-PA group, although they have the same amount of bioactive functional groups and glucose. This difference could be due to the cooperative effect of glucose residue and lysine when placed in the same peptide backbone. Lysine residues create an adhesive environment for cells, and polylysine is widely used to modify glass or plastic surfaces to culture specific cell types that are less prone to adhesion. ${ }^{50,51}$ Glucose group is not present in K-PA/E-PA group, and it is conjugated to glutamic acid containing PA scaffold in E-Glc-PA/K-PA. These differences underline the effect of flanking amino acid in a mixed system on cellular responses. Overall, cell adhesion results show that the adhesive differences stem from the spatial organization of the amino acids in PA nanofiber system.

While other groups were comparable with bare surface, proliferation on the E-Glc-PA/K-PA system was $62 \%$, compared to control group. This arrest in cell proliferation may be an indicator of cellular differentiation, since it has been reported that differentiating stem cells are less likely to proliferate. $^{52-54}$ Upon combination of lipid droplet formation and adipogenic gene marker expression analyses with proliferation data, it is likely that significant arrest of cell division of E-Glc-PA/K-PA scaffold was due to induced adipogenic differentiation of MSCs. In addition, the difference between the cell proliferation rate on K-Glc-PA/E-PA and EGlc-PA/K-PA systems further demonstrates that different spatial organizations of PA nanofibers trigger different cellular responses.

By revealing the multilocular lipid droplets, Nile red staining also indicated that E-Glc-PA/K-PA induces MSC differentiation into brown fat adipocytes, not to the white ones. This conclusion was further confirmed by brown and white fat specific gene expression analyses. Brown character of the adipocytes that differentiated from MSCs was measured by leptin and UCP1 gene expression levels. UCP1 is a mitochondrial protein that dissociates oxidative phosphorylation from energy production, leading to increased thermogenesis. ${ }^{33}$ Brown adipose tissue is specialized for thermogenic energy expenditure, and it maintains animal body temperature with unique expression of UCP1..$^{36,46}$ Discovery of the brown fat in human adults was a recent event, as it was first reported in $2007 .^{20}$ Therefore, studies related to brown fat formation attract a lot of interest. Significant enhancement of UCP1 expression of cells cultured on E-Glc-PA/K-PA shows that these cells differentiate into brown adipocytes. Here, increase in UCP1 expression level has to be emphasized. UCP1 gene expressed by cells on E-Glc-PA/K-PA was 60-fold and 110-fold more than the all other groups on day 7 and day 11, respectively. Real time PCR is a quantitative method, and generally 2- or 3-fold changes in gene expression indicate significant difference between the groups. In this respect, the changes that we observed are remarkably significant. Another fact that needs to be mentioned is the direct relationship between the UCP1 expression increments with the increasing culture period on E-Glc-PA/K-PA scaffold. While UCP1 expression increased 50-fold between day 7 and day 11, remaining groups kept the similar UCP1 expression levels on day 7 and day 11. Taken together with the oil red-O and Nile red staining results, increasing UCP1 expression shows the increased number of brown fat cells committed from MSCs. This conclusion was further supported by leptin gene expression. As noted above, leptin expression in brown fat is lower than in white fat. ${ }^{39}$ qRT-PCR analyses revealed that the least leptin expression was observed in E-Glc-PA/K-PA group. Since all experiments were performed in maintenance medium, the elevation of brown fat gene marker expressions and lipid droplet formations without use of any differentiation medium or agents indicate the potential of E-Glc-PA/K-PA for induction of brown fat differentiation of MSCs.

\section{CONCLUSION}

Early cellular response, lipid droplet formation, and expression levels of adipogenesis marker genes demonstrated that the spatial organization of the functional groups in PA nanofibers determines cellular differentiation profiles of MSCs. In addition, this study introduced a simple, straightforward method to differentiate MSCs to mature adipocytes with brown adipocyte characteristics. As there is no need to use any other differentiation inducing agents, this scaffold eliminates the high cost efforts for obtaining brown fat cells from stem cells. This scaffold system not only can be used for brown fat formation/stem cell differentiation studies but also can be used to obtain brown fat cells from individual's own stem cells with high yield, and these brown fat cells can be used as obesity fighting and thermogenesis agents in future. Overall, the present study shows the importance of spatial organization of bioactive groups of PAs in nanofiber systems and introduced a novel scaffold for brown fat cell production from MSCs.

\section{EXPERIMENTAL SECTION}

Synthesis of Peptide Amphiphile Molecules. The PA molecules were synthesized by standard SPPS (solid phase peptide synthesis) method. The PAs, except E-PA, were constructed on MBHA Rink amide resin, and E-PA [laurylVVAGE] was constructed on Wang resin preloaded with Fmoc$\mathrm{Glu}\left(\mathrm{O}^{t} \mathrm{Bu}\right)$. The resins were swelled in DCM (dichloromethane) for $30 \mathrm{~min}$. Following resin swelling, DCM solvent was exchanged to DMF (dimethylformamide), in which all remaining reactions were carried out. All amino acid couplings, except Fmoc-Ser[-Glc(OAc) $\left.)_{4}\right]-\mathrm{OH}$, were performed with 2 equiv of Fmoc protected amino acid, 1.95 equiv of HBTU, and 3 equiv of $N, N$-diisopropylethylamine (DIEA) in DMF. Coupling duration was at least $3 \mathrm{~h}$ but varied depending on the type of amino acid that was coupled. Fmoc-Ser[Glc$\left.(\mathrm{OAc})_{4}\right]-\mathrm{OH}$ coupling was performed with 1.2 equiv of amino acid, 1.1 equiv of HBTU, and 1.8 equiv of DIEA in DMF. Equivalences are based on the resin that was used for construction. Fmoc removal was performed with $20 \%$ piperidine/DMF solution for $20 \mathrm{~min}$. After each coupling reaction, resin was treated with $10 \%$ acetic anhydride in DMF for $30 \mathrm{~min}$ to block any remaining free amino groups. Before each succeeding event, washing was performed by DMF, DCM, and DMF three times each subsequently. Cleavage of the peptides from resin was carried out with a mixture of trifluoroacetic acid (TFA)/triisopropylsilane (TIS)/water in the ratio of 95:2.5:2.5 for $2 \mathrm{~h}$. Excess TFA was removed by rotary evaporation. The remaining viscous peptide solution was treated with ice-cold diethyl ether overnight at $-20{ }^{\circ} \mathrm{C}$. Ether decantation was performed after centrifugation at $4{ }^{\circ} \mathrm{C}$ and $8000 \mathrm{rpm}$ for $15 \mathrm{~min}$. After complete evaporation of diethyl ether via air drying, the resulting pellet was dissolved in $\mathrm{ddH}_{2} \mathrm{O}$, sonicated for $30 \mathrm{~min}$, freeze-dried at $-80{ }^{\circ} \mathrm{C}$, and lyophilized. Deacetylation of glucose conjugated PAs, which are K-Glc-PA $\left[\right.$ lauryl-VVAGKS $\left[\beta\right.$-D-Glc $\left.(\mathrm{OAc})_{4}\right)$-Am] and E-Glc-PA [Lauryl- 
$\operatorname{VVAGES}\left[\beta\right.$-D-Glc $\left.(\mathrm{OAc})_{4}\right)$-Am $]$, was carried out in solution phase. Acetyl protecting groups on glucose was removed in $\mathrm{NaOMe}$ in methanol solution. The reaction was carried out at room temperature, in argon atmosphere, for 3-4 h by stirring. The reaction was quenched at acidic condition, which is created by a few drops of acetic acid. Solution was removed by rotary evaporator, and the obtained product was dissolved in $\mathrm{ddH}_{2} \mathrm{O}$ and freeze-dried. The peptides were stored at $-20{ }^{\circ} \mathrm{C}$.

Liquid Chromatography-Mass Spectrometry (LCMS). The synthesized peptides were characterized by liquid chromatography-mass spectrometry (LC-MS) on an Agilent 6530 Q-TOF mass spectrometer equipped with ESI source and reverse phase analytical high performance liquid chromatography. Basic conditions and acidic conditions were used to identify negatively charged and positively charged peptide molecules, respectively. For basic conditions, Zorbax ExtendC18 (4.6 $\mathrm{mm} \times 50 \mathrm{~mm})$ column and water/acetonitrile gradient with $0.1 \%$ volume of $\mathrm{NH}_{4} \mathrm{OH}$ were used. For acidic conditions, Zorbax $300 \mathrm{SB}-\mathrm{C} 8(4.6 \mathrm{~mm} \times 100 \mathrm{~mm})$ column and water/acetonitrile gradient with $0.1 \%$ volume of formic acid were used. An Agilent 1200 preparative reverse-phase HPLC system was used for purification of PA molecules. As stationary phase, a Luna $5 \mathrm{u} \mathrm{C8}(2)(21.20 \mathrm{~mm} \times 100 \mathrm{~mm})$ column for acidic conditions and a Gemini 5u C18 (21.20 mm $\times 100 \mathrm{~mm}$ ) column for basic conditions were used to purify positively charged and negatively charged PA molecules, respectively. As mobile phase, water/acetonitrile gradient with $0.1 \% \mathrm{NH}_{4} \mathrm{OH}$ for basic conditions and water/acetonitrile gradient with $0.1 \%$ TFA for acidic conditions were used.

Formation of Self-Assembled PA Nanostructures. To induce nanofiber formation, PA solutions were prepared from the solid form of the PAs by dissolving in $\mathrm{ddH}_{2} \mathrm{O}$. K-GlcPA[lauryl-VVAGKS $(\beta$-D-Glc)-Am ], E-Glc-PA [lauryl$\operatorname{VVAGES}(\beta$-D-Glc)- Am], and K-PA [lauryl-VVAGK-Am] solutions were prepared at $1 \mathrm{mM}$ concentration. E-PA [lauryl-VVAGE] was prepared at $0.5 \mathrm{mM}$ concentration, and PA solutions were sonicated for $15 \mathrm{~min}$ and sterilized under UV for $15 \mathrm{~min}$. PA nanostructures were formed on well plates by mixing counter charged PAs with equal volume. Coated plates were incubated under standard conditions for half an hour to allow homogeneous mixing of PA molecules. After the incubation, plates were left under laminar flow hood overnight to air-dry the peptide gels. Prior to cell seeding, plates were sterilized by UV radiation for $30 \mathrm{~min}$.

Circular Dichroism. Secondary structures of the PA molecules were characterized by J-815 Jasco CD spectrophotometer in the far ultraviolet region. PA solutions were prepared and nanofiber formations were conducted as explained in the "Formation of Self-Assembled PA Nanostructures” part. PA solutions and peptide gel were incubated for 15 min prior to measurement in order to allow self-assembly formation and diluted 1:3 with $\mathrm{ddH}_{2} \mathrm{O}$. CD spectrum was measured from 300 to $190 \mathrm{~nm}$, with $100 \mathrm{~nm} / \mathrm{min}$ scanning speed, 0.1 data pitch, $1 \mathrm{~nm}$ bandwidth, $4 \mathrm{~s}$ DIT, and measurements were performed with three accumulations. Selectivity parameter was selected as standard. Measurements were performed in quartz cuvettes with $1 \mathrm{~mm}$ path length. The results were obtained as degree of ellipticity and converted into molar ellipticity, the unit of $\mathrm{deg} \cdot \mathrm{cm}^{2} \cdot \mathrm{dmol}^{-1}$, using the formula $[\theta]=100 \times \Omega /(C \times 0.1)$ where $\Omega$ is degree of ellipticity, $C$ is the molar concentration, and 0.1 is the cell path length in centimeters.
Scanning Electron Microscopy. For SEM imaging, peptide hydrogels were prepared on cleaned silica wafers by mixing $10 \mathrm{mM}$ PA solutions at 1:1 ratio except combinations including E-PA. PAs that were combined with E-PA were mixed at 2:1 ratio, where E-PA was 1 equiv. Obtained gels were incubated for $20 \mathrm{~min}$ at room temperature to allow gelation and then dehydrated. Dehydration was performed in 20, 40, 60, 80, and $100 \%$ ethanol solutions sequentially. Dehydrated gels were dried with a Tousimis Autosamdri-815B critical-point-drier to preserve the network structure. The dried samples were covered with $\mathrm{Au} / \mathrm{Pd}$ at $5 \mathrm{~nm}$ thickness and visualized under high vacuum with a FEI Quanta 200 FEG scanning electron microscope equipped with ETD detector.

Transmission Electron Microscopy. TEM samples were prepared on a 200-mesh copper TEM grid. Nanostructures were formed as explained above, and these solutions were 10 times diluted. An amount of $30 \mu \mathrm{L}$ of the solutions was dropped on a parafilm, which is a hydrophobic surface, and the grid was placed onto the droplet. After $5 \mathrm{~min}$ of incubation, grid was taken, excess PA solution was removed, and the sample was stained with 2 wt \% uranyl acetate for $3 \mathrm{~min}$. Immediately after 3 min, uranyl acetate was removed and the grid was rinsed with $\mathrm{ddH}_{2} \mathrm{O}$ and left for air drying. TEM images were acquired with FEI Tecnai G2 F30 TEM at $300 \mathrm{kV}$.

Cell Culture and Maintenance. Rat mesenchymal stem cells (MSCs) (Invitrogen) were cultured as monolayer cultures in maintenance medium, DMEM (Dulbecco's modified Eagle medium) supplemented with $10 \%$ FBS and $1 \%$ penicillinstreptomycin, in tissue culture plates under standard conditions (at $37{ }^{\circ} \mathrm{C}$ under $5 \% \mathrm{CO}_{2}$ ). Cells were seeded at $3000-5000$ cells $/ \mathrm{cm}^{2}$ and passaged at $80 \%$ confluency by trypsin-EDTA (ethylenediaminetetraacetic acid) (0.025\%). The culture medium was replaced every 3-4 days. In all experiments, cells between passages 6-9 were used. All cell culture reagents were purchased from Invitrogen.

Viability Assay: MTT Assay. Viability of MSCs seeded on nanofiber networks was quantified by MTT assay (Sigma, catalog no. TOX-1). In order to perform MTT assay, cells at a density of $1.2 \times 10^{4}$ cells $/ \mathrm{cm}^{2}$ were seeded on PA coated wells and on bare wells of 96 well-plates. Then, plates were incubated under standard conditions for $12 \mathrm{~h}$. After incubation period, medium was replaced with phenol red free medium containing (3-[4,5-dimethylthiazol-2-yl]-2,5-diphenyltetrazolium bromide) (MTT) reagent and cells were incubated for $4 \mathrm{~h}$ at standard cell culture conditions. Following the incubation period, plates were removed from the incubator, and the resulting formazan crystals, formed by viable cells through cleavage of tetrazolium ring, were dissolved in MTT solubilization solution [M-8910]. Spectroscopic measurement was performed at a wavelength of $570 \mathrm{~nm}$, and obtained results were converted into \% viability against bare surface.

Cell Adhesion. Adhesion capacity of MSCs on PA coated and on bare surfaces was analyzed after $4 \mathrm{~h}$ of incubation. To perform adhesion test, growth medium of the flask was replaced with adhesion medium, which is serum-free low glucose phenol red including DMEM supplemented with $3 \mathrm{wt} \%$ BSA and 0.05 wt $\%$ cycloheximide, and cells were incubated for $1 \mathrm{~h}$ at standard cell culture conditions. To detach cells from the flask surface, trypsin-EDTA was added and incubated for $30 \mathrm{~s}$. Later $6 \times 10^{3}$ cells $/ \mathrm{cm}^{2}$ were seeded on PA coated wells and on bare wells and incubated under standard conditions for $5 \mathrm{~h}$. After 5 $\mathrm{h}$, the medium was removed by aspiration, the wells were washed with PBS, and cells were stained with $2 \mu \mathrm{M}$ calcein AM 
(Invitrogen) in PBS for $40 \mathrm{~min}$. Stained cells were imaged by an inverted fluorescent microscope and counted using ImageJ software. The experiment was carried out on a 96-well plate.

Cell Proliferation. Proliferation of cells was determined by cell proliferation ELISA, BrdU (colorimetric) (Roche) according to the manufacturer's protocol. A 96-well plate was used for the proliferation assay. Briefly, $1.2 \times 10^{4} \mathrm{cells} / \mathrm{cm}^{2}$ were seeded on PA coated wells and on bare wells and incubated for $48 \mathrm{~h}$ under standard conditions. After $48 \mathrm{~h}$, medium was replaced with $10 \mu \mathrm{M}$ BrdU (bromodeoxyuridine) containing medium and cells were incubated under standard conditions for an additional $4 \mathrm{~h}$. Cells were then fixed and stained by using labeled antibody against BrdU. Cells were washed three times with PBS, and substrate solution was added. After $20 \mathrm{~min}$, color development was measured at a wavelength of $370 \mathrm{~nm}$ by using a microplate reader (Molecular Devices Spectramax M5) and subtracted from reference wavelength (492 $\mathrm{nm})$ values.

Oil Red-O Staining. Oil red-O assay was performed to evaluate deposition of triglycerides and lipids. Nanofiber formation on 96-well plate was done as explained. Cells were seeded at $1.2 \times 10^{4}$ cells $/ \mathrm{cm}^{2}$ density and incubated under standard conditions until the indicated experiment time points. At the indicated time points, medium was discarded by aspiration and cells were washed with $1 \times$ PBS and fixed in $10 \%$ formalin in $1 \times$ PBS. After $10 \mathrm{~min}$, fixation solution was refreshed and the cells were incubated for an additional $1 \mathrm{~h}$ at room temperature. Later, wells were washed with $\mathrm{ddH}_{2} \mathrm{O}$ and incubated in $60 \%$ isopropanol for $5 \mathrm{~min}$ at room temperature. After $5 \mathrm{~min}$, isopropanol was discarded and plate was left open to air-dry. When the wells were completely dried, $100 \mu \mathrm{L}$ oil red-O working solution per well was added and incubated for $10 \mathrm{~min}$ by gently shaking on a shaker. Unbound dyes were washed with $\mathrm{ddH}_{2} \mathrm{O} 4$ times. Finally, $\mathrm{dd}_{2} \mathrm{O}$ was added on wells and images were acquired under an inverted light microscope.

Real-Time Gene Expression Analysis. Gene expression profiles of differentiating MSCs were assessed by quantitative real time PCR (qRT-PCR) analyses. For total RNA extraction, cells were seeded at $1.2 \times 10^{4}$ cells $/ \mathrm{cm}^{2}$ density on PA coated and bare well surfaces. For RNA extraction, cells were grown on 24-well plates. Total RNAs of cells were extracted by using TRIzol (Invitrogen) according to the manufacturer's instructions. Concentration and purity of isolated RNAs were measured by Nanodrop 2000 (Thermoscientific). All samples were diluted to $50 \mathrm{ng} / \mu \mathrm{L}$ concentration prior to their use in qRT-PCR. Conversion of RNAs to cDNA and amplification of cDNAs were performed by using SuperScript III Platinum SYBR Green one-step qRT-PCR kit (Invitrogen) according to the manufacturer's protocol. Reaction conditions were briefly as follows: $55^{\circ} \mathrm{C}$ for $5 \mathrm{~min}, 95^{\circ} \mathrm{C}$ for $5 \mathrm{~min}, 40$ cycles of $95^{\circ} \mathrm{C}$ for $15 \mathrm{~s}, X^{\circ} \mathrm{C}$ (where $X$ is the annealing temperature of the primers; see Table S2) for $30 \mathrm{~s}$ and $40{ }^{\circ} \mathrm{C}$ for $1 \mathrm{~min}$, followed by a melting curve analysis in order to confirm product specificity. The reaction efficiencies for each primer were determined by plotting a standard curve with 10 -fold dilutions of the total RNA. Primary gene expression levels were normalized to GAPDH (a housekeeping gene), and comparative Ct method (Pfaffl method) was used to analyze results.

Nile Red/TO-PRO-3 One Step Staining. Nile red/TOPRO-3 staining was performed to visualize cells under confocal microscope for high resolution analyses of lipid droplets and nucleus. For Nile red/TO-PRO-3 staining, nanofiber formation was carried out with the same method on glass coverslips that are placed in 24-well plates. Cells were seeded at $1.2 \times 10^{4}$ cells $/ \mathrm{cm}^{2}$ density on PA coated and bare glass surfaces and incubated under standard conditions. At the indicated time points, cell medium was discarded, cells were washed with $1 \times$ PBS twice, and cells were fixed with $4 \%$ paraformaldehyde for $20 \mathrm{~min}$. Later, fixative solution was removed, cells were washed with $1 \times \mathrm{PBS}$, and $250 \mu \mathrm{L}$ per well Nile red/TO-PRO-3 solution $(100 \mathrm{ng} / \mathrm{mL}$ Nile red and 1:1000 dilution of TO-PRO3 in $1 \times$ PBS) was added. After a 20 min incubation period, Nile red/TO-PRO-3 solution was discarded and cells were washed with $\mathrm{ddH}_{2} \mathrm{O}$. Coverslips were then removed from wells, mounted with Prolong gold antifade mounting reagent (Invitrogen), and imaged under Carl Zeiss LSM510 confocal microscope.

\section{ASSOCIATED CONTENT}

\section{S Supporting Information}

The Supporting Information is available free of charge on the ACS Publications website at DOI: 10.1021/acs.bioconjchem.6b00632.

LC-MS results of PAs, CD result of PA molecules, oil red-O staining images at day 11 , additional confocal microscope images of cells stained with Nile red/TOPRO-3, molecular weight of PAs, list of primer sequences used for qRT-PCR experiments (PDF)

\section{AUTHOR INFORMATION}

\section{Corresponding Authors}

*E-mail: atekinay@bilkent.edu.tr.

*E-mail: moguler@unam.bilkent.edu.tr. Fax: +90 312266 4365. Phone: +90 3122908985.

ORCID

Ayse B. Tekinay: 0000-0002-4453-814X

Mustafa O. Guler: 0000-0003-1168-202X

\section{Notes}

The authors declare no competing financial interest.

\section{ACKNOWLEDGMENTS}

We thank Z. Erdogan and M. Guler for technical help. This work was partially funded by TUBITAK (Grant 113T045) and TUBA. O.S.C. is supported by the TUBITAK-BIDEB 2210-C MS.

\section{ABBREVIATIONS}

PA, peptide amphiphile; MSC, mesenchymal stem cell; SEM, scanning electron microscopy; TEM, transmission electron microscopy; CD, circular dichroism; HPLC, high performance liquid chromatography; LC-MS, liquid chromatography-mass spectrometry; ECM, extracellular matrix; FABP4, fatty acid binding protein 4 ; UCP1, uncoupling protein 1

\section{REFERENCES}

(1) Sun, C., Marcello, M., Li, Y., Mason, D., Lévy, R., and Fernig, D. G. (2016) Selectivity in glycosaminoglycan binding dictates the distribution and diffusion of fibroblast growth factors in the pericellular matrix. Open Biol. 6, 1-17.

(2) Koo, L. Y., Irvine, D. J., Mayes, A. M., Lauffenburger, D. a, and Griffith, L. G. (2002) Co-regulation of cell adhesion by nanoscale RGD organization and mechanical stimulus. J. Cell Sci. 115, 14231433. 
(3) Cui, H., Webber, M. J., and Stupp, S. I. (2010) Self-assembly of peptide amphiphiles: from molecules to nanostructures to biomaterials. Biopolymers 94, 1-18.

(4) Hartgerink, J. D., Beniash, E., and Stupp, S. I. (2002) Peptideamphiphile nanofibers: a versatile scaffold for the preparation of selfassembling materials. Proc. Natl. Acad. Sci. U. S. A. 99, 5133-5138.

(5) Boekhoven, J., Zha, R. H., Tantakitti, F., Zhuang, E., Zandi, R., Newcomb, C. J., and Stupp, S. I. (2015) Alginate-peptide amphiphile core-shell microparticles as a targeted drug delivery system. RSC $A d v$. $5,8753-8756$.

(6) Fletcher, J. M., Harniman, R. L., Barnes, F. R. H., Boyle, A. L., Collins, A., Mantell, J., Sharp, T. H., Antognozzi, M., Booth, P. J., Linden, et al. (2013) Self-Assembling Cages from Coiled-Coil Peptide Modules. Science 340, 595-599.

(7) Ustun Yaylaci, S., Sardan Ekiz, M., Arslan, E., Can, N., Kilic, E., Ozkan, H., Orujalipoor, I., Ide, S., Tekinay, A. B., and Guler, M. O. (2016) Supramolecular GAG-like Self-Assembled Glycopeptide Nanofibers Induce Chondrogenesis and Cartilage Regeneration. Biomacromolecules 17, 679-689.

(8) Gulseren, G., Yasa, I. C., Ustahuseyin, O., Tekin, E. D., Tekinay, A. B., and Guler, M. O. (2015) Alkaline Phosphatase-Mimicking Peptide Nanofibers for Osteogenic Differentiation. Biomacromolecules 16, 2198-2208.

(9) Goktas, M., Cinar, G., Orujalipoor, I., Ide, S., Tekinay, A. B., and Guler, M. O. (2015) Self-Assembled Peptide Amphiphile Nanofibers and PEG Composite Hydrogels as Tunable ECM Mimetic Microenvironment. Biomacromolecules 16, 1247-1258.

(10) Sardan, M., Kilinc, M., Genc, R., Tekinay, A. B., and Guler, M. O. (2013) Cell penetrating peptide amphiphile integrated liposomal systems for enhanced delivery of anticancer drugs to tumor cells. Faraday Discuss. 166, 269.

(11) Mammadov, R., Mammadov, B., Guler, M. O., and Tekinay, A. B. (2012) Growth factor binding on heparin mimetic peptide nanofibers. Biomacromolecules 13, 3311-3319.

(12) Toksoz, S., Mammadov, R., Tekinay, A. B., and Guler, M. O. (2011) Electrostatic effects on nanofiber formation of self-assembling peptide amphiphiles. J. Colloid Interface Sci. 356, 131-137.

(13) Hamley, I. W., Dehsorkhi, A., and Castelletto, V. (2013) Coassembly in Binary Mixtures of Peptide Amphiphiles Containing Oppositely Charged Residues. Langmuir 29, 5050-5059.

(14) Dehsorkhi, A., Castelletto, V., and Hamley, I. W. (2014) Selfassembling amphiphilic peptides. J. Pept. Sci. 20, 453-467.

(15) Toksoz, S., and Guler, M. O. (2009) Self-assembled peptidic nanostructures. Nano Today 4, 458-469.

(16) Harrington, D. A., Cheng, E. Y., Guler, M. O., Lee, L. K., Donovan, J. L., Claussen, R. C., and Stupp, S. I. (2006) Branched peptide-amphiphiles as self-assembling coatings for tissue engineering scaffolds. J. Biomed. Mater. Res., Part A 78A, 157-167.

(17) Pratt, M. R., and Bertozzi, C. R. (2005) Synthetic glycopeptides and glycoproteins as tools for biology. Chem. Soc. Rev. 34, 58-68.

(18) Westerlind, U. (2012) Synthetic glycopeptides and glycoproteins with applications in biological research. Beilstein J. Org. Chem. 8, 804-818.

(19) Sasisekharan, R., Raman, R., and Prabhakar, V. (2006) Glycomics Approach To Structure-Function Relationships of Glycosaminoglycans. Annu. Rev. Biomed. Eng. 8, 181-231.

(20) Hassan, M., Latif, N., and Yacoub, M. (2012) Adipose tissue: friend or foe? Nat. Rev. Cardiol. 9, 689-702.

(21) Kotz, J. (2012) Browning fat. SciBX 5, 18-20.

(22) Greenfield, N. J. (2007) Using circular dichroism spectra to estimate protein secondary structure. Nat. Protoc. 1, 2876-2890.

(23) Menssen, A., Häupl, T., Sittinger, M., Delorme, B., Charbord, P., and Ringe, J. (2011) Differential gene expression profiling of human bone marrow-derived mesenchymal stem cells during adipogenic development. BMC Genomics 12, 461.

(24) Mehlem, A., Hagberg, C. E., Muhl, L., Eriksson, U., and Falkevall, A. (2013) Imaging of neutral lipids by oil red $O$ for analyzing the metabolic status in health and disease. Nat. Protoc. 8, 1149-1154.
(25) Ramirez-Zacarias, J. L., Castro-Mufiozledo, F., and KuriHarcuch, W. (1992) Quantitation of adipose conversion and triglycerides by staining intracytoplasmic lipids with Oil red O. Histochemistry 97, 493-497.

(26) Chandran, M., Phillips, S. A., Ciaraldi, T., and Henry, R. R. (2003) Adiponectin: More Than Just Another Fat Cell Hormone? Diabetes Care 26, 2442-2450.

(27) Pajvani, U. B., and Scherer, P. E. (2003) Adiponectin: systemic contributor to insulin sensitivity. Curr. Diabetes Rep. 3, 207-213.

(28) Kiess, W., Petzold, S., Töpfer, M., Garten, A., Blüher, S., Kapellen, T., Körner, A., and Kratzsch, J. (2008) Adipocytes and adipose tissue. Best Pract. Res. Clin. Endocrinol. Metab. 22, 135-153.

(29) Valan Arasu, M., Ilavenil, S., Kim, D. H., Gun Roh, S., Lee, J.-C., and Choi, K. C. (2014) In Vitro and In Vivo Enhancement of Adipogenesis by Italian Ryegrass (Lolium multiflorum) in 3T3-L1 Cells and Mice. PLoS One 9, e85297.

(30) Hotamisligil, G. S., and Bernlohr, D. a. (2015) Metabolic functions of FABPs-mechanisms and therapeutic implications. Nat. Rev. Endocrinol. 11, 592-605.

(31) Lowe, C. E., O'Rahilly, S., and Rochford, J. J. (2011) Adipogenesis at a glance. J. Cell Sci. 124, 2681-2686.

(32) Urs, S., Smith, C., Campbell, B., Saxton, A. M., Taylor, J., Zhang, B., Snoddy, J., Jones Voy, B., and Moustaid-Moussa, N. (2004) Gene Expression Profiling in Human Preadipocytes and Adipocytes by Microarray Analysis. J. Nutr. 134, 762-770.

(33) Cristancho, A. G., and Lazar, M. a. (2011) Forming functional fat: a growing understanding of adipocyte differentiation. Nat. Rev. Mol. Cell Biol. 12, 722-34.

(34) Moreno-Navarrete, J. M., and Fernández-Real, J. M. (2012) Adipocyte Differentiation. Adipose Tissue Biol., 17-38.

(35) Ali, A. T., Hochfeld, W. E., Myburgh, R. and Pepper, M. S. (2013) Adipocyte and adipogenesis. Eur. J. Cell Biol. 92, 229-236.

(36) Yu, J., Zhang, S., Cui, L., Wang, W., Na, H., Zhu, X., Li, L., Xu, G., Yang, F., Christian, M., and Liu, P. (2015) Lipid droplet remodeling and interaction with mitochondria in mouse brown adipose tissue during cold treatment. Biochim. Biophys. Acta, Mol. Cell Res. 1853, 918-928.

(37) Greenspan, P., Mayer, E. P., and Fowler, S. D. (1985) Nile Red a Selective Fluorescent Stain for Intracellular Lipid Droplets. J. Cell Biol. 100, 965-973.

(38) Van Hooijdonk, C. A. E. M., Glade, C. P., and Van Erp, P. E. J. (1994) TO-PRO-3 iodide: A novel HeNe laser-excitable DNA stain as an alternative for propidium iodide in multiparameter flow cytometry. Cytometry 17, 185-189.

(39) Cinti, S., Frederich, R. C., Zingaretti, M. C., De Matteis, R, Flier, J. S., and Lowell, B. B. (1997) Immunohistochemical localization of leptin and uncoupling protein in white and brown adipose tissue. Endocrinology 138, 797-804.

(40) Saely, C. H., Geiger, K., and Drexel, H. (2012) Brown versus white adipose tissue: A mini-review. Gerontology 58, 15-23.

(41) Enerback, S. (2010) Human Brown Adipose Tissue. Cell Metab. $11,248-252$.

(42) Morganstein, D. L., Wu, P., Mane, M. R., Fisk, N. M., White, R., and Parker, M. G. (2010) Human fetal mesenchymal stem cells differentiate into brown and white adipocytes: a role for ERRalpha in human UCP1 expression. Cell Res. 20, 434-444.

(43) Sharp, L. Z., Shinoda, K., Ohno, H., Scheel, D. W., Tomoda, E., Ruiz, L., Hu, H., Wang, L., Pavlova, Z., Gilsanz, V., and Kajimura, S. (2012) Human BAT Possesses Molecular Signatures That Resemble Beige/Brite Cells. PLoS One 7, e49452.

(44) Wu, J., Bostrom, P., Sparks, L. M., Ye, L., Choi, J. H., Giang, A. H., Khandekar, M., Virtanen, K. a., Nuutila, P., Schaart, G., et al. (2012) Beige adipocytes are a distinct type of thermogenic fat cell in mouse and human. Cell 150, 366-376.

(45) Ahfeldt, T., Schinzel, R. T., Lee, Y.-K., Hendrickson, D., Kaplan, A., Lum, D. H., Camahort, R., Xia, F., Shay, J., Rhee, E. P., et al. (2012) Programming human pluripotent stem cells into white and brown adipocytes. Nat. Cell Biol. 14, 209-219. 
(46) Schulz, T. J., Huang, T. L., Tran, T. T., Zhang, H., Townsend, K. L., Shadrach, J. L., Cerletti, M., McDougall, L. E., Giorgadze, N., Tchkonia, T., et al. (2011) Identification of inducible brown adipocyte progenitors residing in skeletal muscle and white fat. Proc. Natl. Acad. Sci. U. S. A. 108, 143-8.

(47) Sethi, J. K., and Vidal-Puig, A. J. (2007) Adipose tissue function and plasticity orchestrate nutritional adaptation. J. Lipid Res. 48, 125362.

(48) Ussar, S., Lee, K. Y., Dankel, S. N., Boucher, J., Haering, M.-F., Kleinridders, A., Thomou, T., Xue, R., Macotela, Y., Cypess, A. M., et al. (2014) ASC-1, PAT2, and P2RX5 are cell surface markers for white, beige, and brown adipocytes. Sci. Transl. Med. 6, 247ra103.

(49) Stendahl, J. C., Rao, M. S., Guler, M. O., and Stupp, S. I. (2006) Intermolecular forces in the self-assembly of peptide amphiphile nanofibers. Adv. Funct. Mater. 16, 499-508.

(50) Ando, T., and Yonamoto, Y. (2015) In Situ EPR Detection of Reactive Oxygen Species in Adherent Cells Using Polylysine-Coated Glass Plate. Appl. Magn. Reson. 46, 977.

(51) Yavin, E., and Yavin, Z. (1974) Attachment and Culture of Dissociated Cells from Rat Embryo Cerebral Hemispheres on Polylysine-Coated Surface. J. Cell Biol. 62, 540-546.

(52) Boriani, F., Warr, R., and Ascione, R. (2011) Adipose Stem Cells and Regenerative Medicine (Illouz, Y.-G., and Sterodimas, A., Eds.) Springer Science \& Business Media.

(53) Cooper, G. M. (2000) The Cell: A Molecular Approach, 2nd ed. Sinauer Associates.

(54) Minoo, P., Sullivan, W., Solomon, L. R., Martin, T. E., Toft, D. O., and Scott, R. E. (1989) Loss of proliferative potential during terminal differentiation coincides with the decreased abundance of a subset of heterogeneous ribonuclear proteins. J. Cell Biol. 109, 19371946. 